Copyright 2004 IEEE — Published in the Proceedings of the 2004 IEEE International Symposium on Biomedical Imaging (ISBI 2004), 15-18 April 2004, Arlington, VA, USA. Personal use of this material is permitted. However, permission to reprint/republish this material for advertising or promotional purposes or for creating new collective works for resale or redistribution to servers or lists, or to reuse any copyrighted component of this work in other works, must be obtained from the IEEE. Contact: Manager, Copyrights and Permissions / IEEE Service Center / 445 Hoes Lane / P.O. Box 1331 / Piscataway, NJ 08855-1331, USA. Telephone: + Intl. 908-562-3966.

\title{
QUANTITATIVE ANALYSIS OF CIRCUMFERENTIAL PLAQUE DISTRIBUTION IN HUMAN CORONARY ARTERIES IN RELATION TO LOCAL VESSEL CURVATURE
}

\author{
Andreas Wahle, ${ }^{1 *}$ Mark E. Olszewski, ${ }^{1}$ Sarah C. Vigmostad, ${ }^{2}$ Rubén Medina,${ }^{4}$ A. Ümit Coşkun,${ }^{5}$ \\ Charles L. Feldman, ${ }^{6}$ Peter H. Stone,${ }^{6}$ Kathleen C. Braddy, ${ }^{3}$ Theresa M. H. Brennan, ${ }^{3}$ \\ James D. Rossen, ${ }^{3}$ Krishnan B. Chandran, ${ }^{2}$ and Milan Sonk ${ }^{1}$ \\ ${ }^{1}$ The University of Iowa, Electrical and Computer Engineering, Iowa City, IA \\ ${ }^{2}$ The University of Iowa, Biomedical Engineering, Iowa City, IA \\ ${ }^{3}$ The University of Iowa, Internal Medicine, Iowa City, IA \\ ${ }^{4}$ Universidad de Los Andes, Facultad de Ingeniería, Mérida, Venezuela \\ ${ }^{5}$ Northeastern University, Mechanical, Industrial and Manufacturing Engineering, Boston, MA \\ ${ }^{6}$ Brigham \& Women's Hospital, Cardiovascular Division, Boston, MA
}

\begin{abstract}
A common hypothesis is that plaque accumulation in curved vessels is biased towards the inner bend of the curvature rather than the outer bend of the curvature. This bias in circumferential plaque distribution is likely associated with lower wall shear stress on the inner bend of the curved vessel. We quantitatively analyzed this effect in a set of 37 in-vivo human coronary artery segments from 31 patients. Three-dimensional models of the arteries were generated by an established system for fusion of image data from Xray angiography and intravascular ultrasound. Our results showed that the hypothesis held in the majority of vessels $(p<0.001)$, and that the effect increases with curvature. However, no evidence could be found for a direct relationship between plaque distribution and curvature in complex vessel geometries, thus motivating a more detailed analysis of wall shear stress patterns and their impact on circumferential plaque distribution.
\end{abstract}

\section{INTRODUCTION}

The progression of cardiovascular disease as a function of local hemodynamics and plaque morphology in human vasculature is of increasing interest [1,2]. As is frequently observed, plaque is not distributed homogeneously and tends to be circumferentially asymmetric (Fig. 1). The purpose of this study was to evaluate if there is a direct relationship between the circumferential plaque distribution and lo-

Supported in part by grant R01 HL63373 of the National Institutes of Health; by grant BES 9902011 of the National Science Foundation; and by AstraZeneca; Dr. Medina was supported by the Universidad de Los Andes and FONACIT, Venezuela. _ * E-mail: a.wahle@ieee.org cal vessel curvature. We have developed a comprehensive system for the fusion of images from biplane angiography and intravascular ultrasound (IVUS), resulting in geometrically correct 3-D or 4-D (3-D + time) models of a vessel (Fig. 2). These models can be utilized for accurate hemodynamic and morphologic analyses [2-5]. The local wall shear stress tends to be lower on the inner bend of a curved vessel ("inner curvature") than on the outer bend ("outer curvature") $[2,6]$. Therefore, plaque in native coronary arteries should accumulate more on the inner curvature of a vessel segment as compared to the outer curvature of the vessel. This hypothesis can frequently be confirmed visually in the reconstructed 3-D models obtained from fusion (Fig. 3). In the following sections, we will present a quantification of this effect in a set of patients from the University of Iowa Hospitals \& Clinics and Brigham \& Women's Hospital.

\section{METHODS}

The basis of this analysis is the previously developed fusion system that utilizes X-ray angiography and IVUS to generate a geometrically correct 3-D model of a vessel segment. This system is described in detail elsewhere [2-4], thus only a brief overview of it will be given. This paper focuses on the determination of local plaque thickness, curvature indices, and the classification of vessel regions that either confirm or do not confirm the given hypothesis.

\subsection{3-D fusion}

The pullback path of the IVUS catheter as well as the vessel lumen outline are extracted from the angiograms (Fig. 3). 


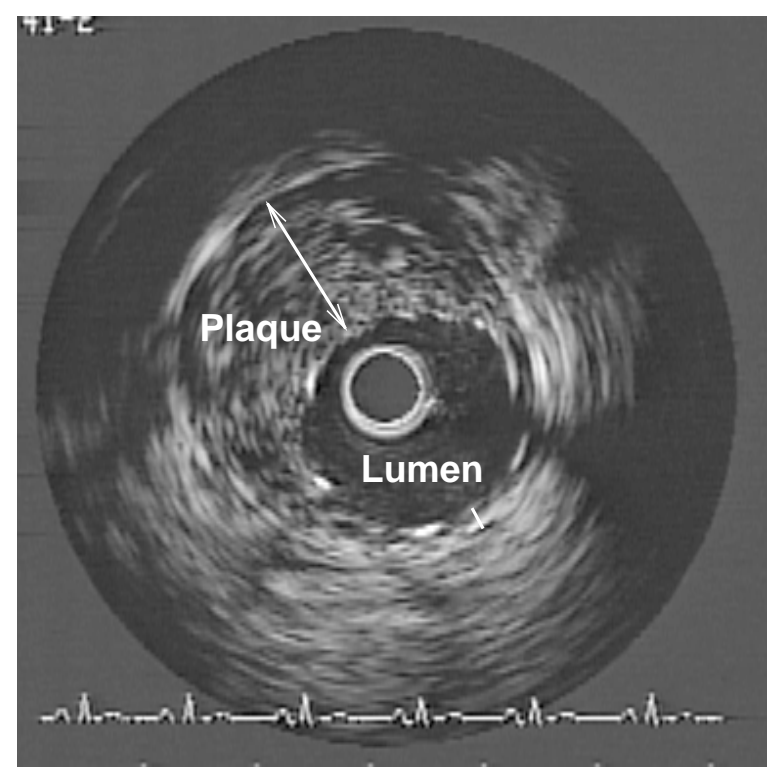

Fig. 1. This IVUS frame from an in-vivo human left anterior descending artery shows the asymmetry in circumferential plaque distribution, but does not give any information about the axial orientation of the frame.

Accurate cross-sectional information is obtained by segmentation of the lumen/plaque and media/adventitia contours from IVUS. The desired 3-D model is derived by mapping the segmented IVUS contours into 3-D space according to the 3-D reconstructed pullback trajectory, and by using the angiographic vessel outline as a reference for the absolute orientation of the IVUS frames [3]. For this study, only frames of the end-diastolic phase were used. The cross sections of the 3-D model are oriented perpendicularly to the pullback path of the IVUS catheter, which does not necessarily correspond with the vessel centerline. Therefore, the contours are resampled according to the centroids of the media/adventitia contours in a post-processing step. In each frame, the centroids are calculated for both lumen/plaque and media/adventitia contours. Connecting the media/adventitia centroids of all frames yields the centerline of the vessel that is used to perpendicularly resample the contours, thus ensuring that plaque-thickness and curvature analyses are not distorted by the non-orthogonal orientation of the IVUS catheter.

\subsection{Local plaque thickness and curvature indices}

To obtain the plaque thickness from the two 3-D contours, radial lines are used that connect the vessel centroid to each contour point in each frame (Fig. 1). Instead of the absolute thickness values, the plaque distribution is expressed relative to the average plaque thickness within each frame. Thus, the resulting values reflect the circumferential plaque
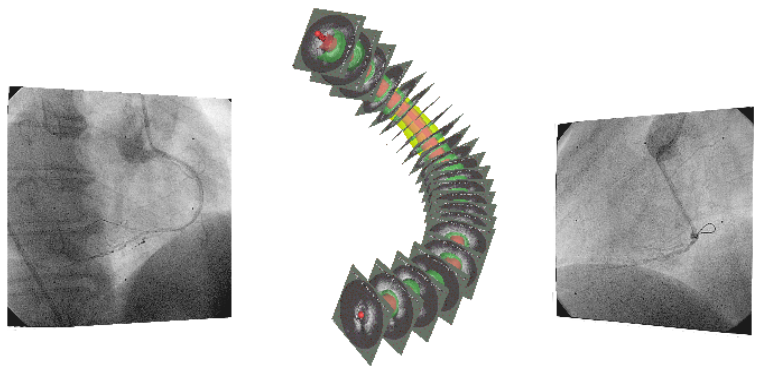

Fig. 2. Fusion between biplane angiography and IVUS creates a 3-D model of the lumen and adventitia surfaces by placing the IVUS frames in their geometrically correct location and orientation.
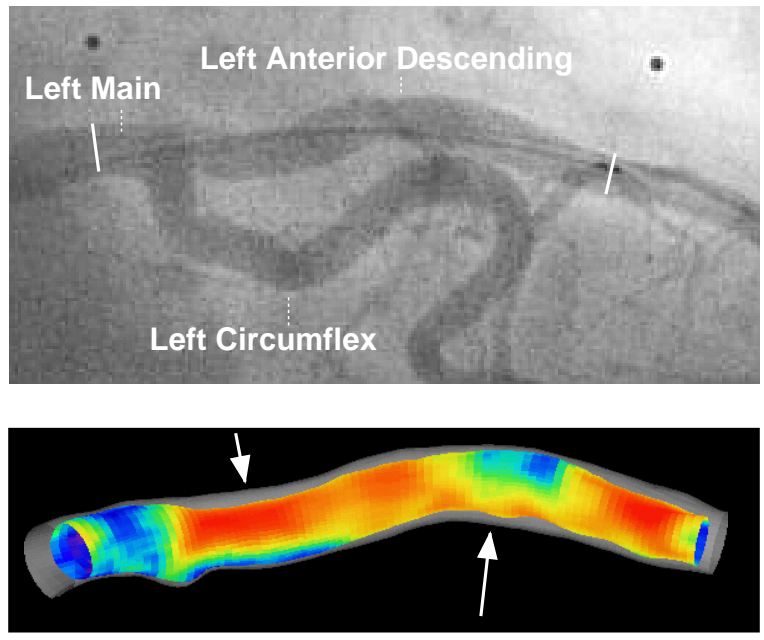

Fig. 3. The angiogram depicts the lumen of this left coronary artery, with the IVUS catheter inserted, and the lines marking the range of the pullback; below the reconstructed 3-D model showing the two surfaces and the plaque thickness as color coding, the arrows mark plaque accumulations at the inner curvatures.

distribution within each frame. All vertices (here: pairs of lumen and adventitia border points) are annotated with their respective absolute and circumferential plaque thickness.

The definition of a local curvature index consists of two steps: (1) determining the local curvature at each frame; (2) determining the curvature indices for each circumferential point within that frame. Differential geometry delivers a set of three orthonormal unit vectors (Frenet frame [3]) for each point on a curved line: $\vec{t}$ (tangent); $\vec{n}$ (normal); and $\vec{b}$ (binormal). The curvature $\kappa$ and the torsion $\tau$ are the angular velocities of the vectors $\vec{t}$ and $\vec{b}$, respectively. Since vessel torsion is not considered here, we are mainly interested in the curvature $\kappa$ and the direction of the curvature indicated by its normal $\vec{n}$. With $\vec{c}(s)$ specifying the point $[x(s), y(s), z(s)]$ of centerline $c$ at location $s$, 


$$
\begin{aligned}
\vec{c}^{\prime \prime}(s) & =\vec{t}^{\prime}(s) \\
& =\kappa(s) \vec{n}(s) \\
\Longrightarrow \quad \kappa(s) & =\left\|\vec{c}^{\prime \prime}(s)\right\| \\
\vec{n}(s) & =\vec{c}^{\prime \prime}(s) / \kappa(s)
\end{aligned}
$$

where the tangent $\vec{t}(s)=\vec{c}^{\prime}(s)$ is the first derivative and $\vec{c}^{\prime \prime}(s)$ is the second derivative of $c$ over the arc length $s$. The normal vector $\vec{n}(s)$ always points towards the origin of the radius of curvature, thus indicating the inner curvature on the circumference. Complementarily, $\kappa(s)$ is a measure of the magnitude of the curvature. To combine the magnitude and direction of the curvature, thereby differentiating between inner and outer curvature, we defined a scalar curvature index $\kappa_{\mathrm{idx}}(s, i)$ for each point $i$ at the frame location $s$ (Fig. 4). A positive $\kappa_{\mathrm{idx}}(s, i)$ indicates inner curvature, a negative index outer curvature, and an index close to zero applies for points on the sides of the curved vessel segment, i.e., perpendicular to $\vec{n}(s)$. The maximum (most positive) curvature index and the minimum (most negative) curvature index depend directly on the magnitude of the curvature $\kappa(s)$ for that frame. The circumferential position of the vertex point indicating the inner curvature is obtained by projecting the unit normal vector $\vec{n}(s)$ on the respective frame $s$. To determine the circumferential position of the $i$ th point relative to the inner curvature reference point, a vector $\vec{v}(s, i)$ is defined from the centroid $\vec{c}(s)$ to the $i$-th point of the lumen contour $\vec{f}(s, i)$ of the IVUS frame at location $s$. Finally, $\kappa_{\mathrm{idx}}(s, i)$ can be obtained using the dot product:

$$
\begin{aligned}
\vec{v}(s, i) & =\vec{f}(s, i)-\vec{c}(s) \\
\kappa_{\mathrm{idx}}(s, i) & =\kappa(s) \vec{n}(s) \cdot\left(\frac{\vec{v}(s, i)}{\|\vec{v}(s, i)\|}\right)
\end{aligned}
$$

Differential geometry assumes that $c$ is a continuous and unit-speed curve, which is not the case. Instead, the 3-D model that was created during the fusion step is discrete in both radial and longitudinal dimensions. Thus, a discretization needs to be taken into account, and a discrete version of the Frenet-Serret formulas was utilized. Also, smoothing was applied for both the 3-D models and for the calculation of the normal vectors $\vec{n}$. A comprehensive discussion on the determination of curvature and torsion in vascular structures is presented in [7].

\subsection{Combining plaque thickness and curvature indices}

To categorize each vertex point according to its respective plaque thickness and curvature index, four regions $R_{x y}$ were defined. The subscript $x$ distinguishes between $a$ ("above average") and $b$ ("below average") for the relative plaque thickness, whereas $y$ is set to $i$ ("inner curvature") for $\kappa_{\mathrm{idx}}(s, i) \geq 0$ and to $o$ ("outer curvature") for

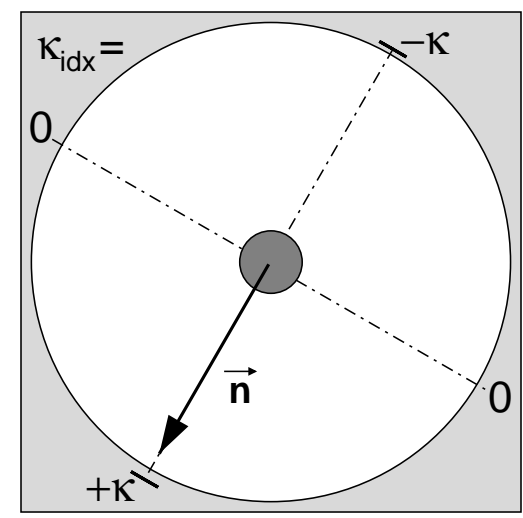

Fig. 4. Definition of the circumferential curvature index $\kappa_{\mathrm{idx}}$ derived from the local curvature $\kappa$ and the projected normal vector $\vec{n}$ for a specific IVUS frame.

$\kappa_{\mathrm{idx}}(s, i)<0$. Thus, four regions $R_{a i}, R_{a o}, R_{b i}$, and $R_{b o}$ are obtained. It is also necessary to define a fifth region $R_{n}$ for "neutral" to indicate regions of low curvature that are excluded to prevent incorporating small perturbations in the results. A vertex point is categorized as $R_{n}$ if its curvature index is below a specified curvature threshold $T$ (i.e., $\left.\left|\kappa_{\mathrm{idx}}(s, i)\right|<T\right)$.

For the hypothesis to hold, the combination of the $R_{a i}$ and $R_{b o}$ should outweigh the combination of $R_{a o}$ and $R_{b i}$, thus implying that plaque is accumulating more at the inner curvature and less at the outer curvature. A ratio $r$ is calculated to indicate the relative number of vertices that satisfy the hypothesis and which are not in the "neutral" region $R_{n}$ :

$$
r=\frac{\left\|R_{a i}+R_{b o}\right\|}{\left\|R_{a i}+R_{a o}+R_{b i}+R_{b o}\right\|}
$$

with $r \geq 0.5$ implying that $\left\|R_{a i}+R_{b o}\right\| \geq\left\|R_{a o}+R_{b i}\right\|$.

\section{RESULTS}

The analysis was performed on 39 in-vivo pullbacks in 37 human coronary vessel segments from 31 patients that were routinely imaged at the University of Iowa Hospitals and Clinics or Brigham \& Women's Hospital. The fusion process described in Section 2.1 was performed for 14 right coronary arteries, 13 left anterior descending arteries, and 10 left circumflex arteries, with pullback lengths ranging from $41.9 \mathrm{~mm}$ to $122.5 \mathrm{~mm}$. The ratio $r$ was determined for 12 different curvature thresholds $T$, which were empirically selected as a function of the maximum curvature over all pullbacks. Thus, $468 r$-values were calculated in total. Over 39 pullbacks, $10.2 \%$ (for $T=2.31 \%$ cm) to $78.3 \%$ (for $\left.T=22.94^{\circ} / \mathrm{cm}\right)$ of the vertex points were excluded and assigned to the region $R_{n}$. Furthermore, vessel portions located in branches, or areas for which strong calcifications 


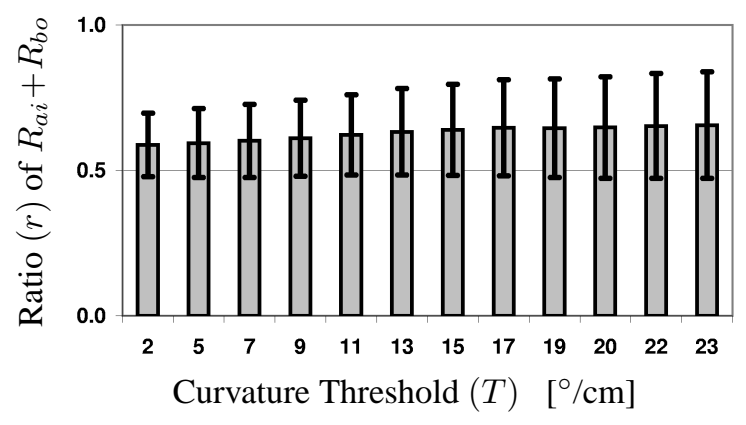

Fig. 5. With increasing curvature threshold $T$, the ratio $r$ of vertices for which the hypothesis holds increases as well; the standard deviation (bars) also increases with $T$.

or dense plaque prevented an accurate segmentation of the borders, were excluded from the analysis. Separate sets of calculations were performed, in which stented areas were either included in or excluded from the analysis.

The hypothesis held for $r \geq 0.5$ in 367 (78.4\%) of the 468 calculations with stented areas included, and in 349 (74.6\%) of 468 calculations with stented areas excluded. In 29 of 37 vessel segments, the hypothesis held for at least half of the thresholds (i.e., $r \geq 0.5$ was true for at least 6 of the $12 T$-values for each of these vessel segments). In 6 of 37 vessel segments (16.2\%), $r \geq 0.5$ was not satisfied for any threshold $T$. Consistently, $r \geq 0.5$ held for the averages over all vessel segments, which is statistically significant for all $T(p<0.001)$, and $r$ increased along with $T$ (Fig. 5). Another observation is that the standard deviation of $r$ over all 39 pullbacks increased from $0.109(T=2.31 \% \mathrm{~cm})$ to 0.183 ( $T=22.94 \% \mathrm{~cm}$, with stents), in accordance with the observation that $r$ frequently decreases with increasing $T$ in segments with overall low $r$-ratios. The comparison of the $r$ value distribution in calculations that included stented areas vs. those in which stented areas were excluded showed no statistical difference $\left(p>0.20\right.$ for $\left.T \geq 6.86^{\circ} / \mathrm{cm}\right)$.

\section{DISCUSSION AND CONCLUSIONS}

The presented study suggests that the observation of circumferential plaque distribution being biased towards the inner curvature of a coronary artery is valid in the majority of vessels, with the limitation of a relatively small number of subjects ( 37 patients) analyzed thus far. These results are in accordance with in-vitro experiments in curved tubes $[2,6]$ and confirm previous studies on a subset of 12 patients [8].

It seems likely that the results depend on the artery being examined. For example, values of $r>0.9$ were obtained in only 6 vessel segments, all of which were left anterior descending arteries. As compared to the other main arteries, this artery is usually less tortuous and has a relatively con- stant curvature. In contrast, pullbacks starting in secondary branches (such as diagonal or obtuse marginal vessels) with small diameter consistently measured $0.3<r<0.6$.

While the hypothesis in general holds in vessels with overall smooth curvature, it fails in more complex vessel geometries with high non-planar tortuosity. In these vessels, blood flow patterns (and therefore shear-stress distributions) are quite complex and no longer correspond to the simple curved-tube model. Therefore, it is essential to include shear-stress calculations in further analyses. We have shown that wall shear stress can be calculated from the 3$\mathrm{D}$ arterial models obtained from fusion, and that they can be performed with high reproducibility [2,5]. Therefore, an extension of the curvature/plaque-thickness analysis by wall shear stress seems feasible and is currently in progress.

\section{REFERENCES}

[1] C. M. Gibson, L. Diaz, K. Kandarpa, F. M. Sacks, R. C. Pasternak, T. Sandor, C. L. Feldman, and P. H. Stone, "Relation of vessel wall shear stress to atherosclerosis progression in human coronary arteries," Arteriosclerosis and Thrombosis, vol. 13, no. 2, pp. 310-315, Feb. 1993.

[2] A. Wahle, S. C. Mitchell, S. D. Ramaswamy, K. B. Chandran, and M. Sonka, "Four-dimensional coronary morphology and computational hemodynamics," in Medical Imaging 2001: Image Processing, M. Sonka and K. M. Hanson, Eds., Bellingham WA, 2001, vol. 4322, pp. 743-754, SPIE Proceedings.

[3] A. Wahle, G. P. M. Prause, S. C. DeJong, and M. Sonka, "Geometrically correct 3-D reconstruction of intravascular ultrasound images by fusion with biplane angiography - methods and validation," IEEE Transactions on Medical Imaging, vol. 18, no. 8, pp. 686-699, Aug. 1999.

[4] M. E. Olszewski, A. Wahle, R. Medina, S. C. Mitchell, and M. Sonka, "Integrated system for quantitative analysis of coronary plaque via data fusion of biplane angiography and intravascular ultrasound," in Computer Assisted Radiology and Surgery (CARS 2003), H. U. Lemke, K. Inamura, M. W. Vannier, A. G. Farman, K. Doi, and J. H. C. Reiber, Eds., Amsterdam, 2003, vol. 1256 of Excerpta Medica International Congress Series, pp. 1117-1122, Elsevier.

[5] A. Ü. Coşkun, Y. Yeghiazarians, S. Kinlay, M. E. Clark, O. J. Ilegbusi, A. Wahle, M. Sonka, J. J. Popma, R. E. Kuntz, C. L. Feldman, and P. H. Stone, "Reproducibility of coronary lumen, plaque, and vessel wall reconstruction and of endothelial shear stress measurements invivo in humans," Catheterization and Cardiovascular Interventions, vol. 60, no. 1, pp. 67-78, Sept. 2003.

[6] A. Santamarina, E. Weydahl, J. M. Siegel, and J. E. Moore, "Computational analysis of flow in a curved tube model of the coronary arteries: Effects of time-varying curvature," Annals of Biomedical Engineering, vol. 26, no. 6, pp. 944-954, Nov. 1998.

[7] R. Medina, A. Wahle, M. E. Olszewski, and M. Sonka, "Curvature and torsion estimation for coronary artery motion analysis," in Medical Imaging 2004: Physiology, Function, and Structure from Medical Images, A. A. Amini and A. Manduca, Eds., Bellingham WA, 2004, vol. 5369, SPIE Proceedings, (in press).

[8] A. Wahle, R. Medina, K. C. Braddy, J. M. Fox, T. M. H. Brennan, J. J. Lopez, J. D. Rossen, and M. Sonka, "Impact of local vessel curvature on the circumferential plaque distribution in coronary arteries," in Medical Imaging 2003: Physiology and Function: Methods, Systems, and Applications, A. V. Clough and A. A. Amini, Eds., Bellingham WA, 2003, vol. 5031, pp. 204-213, SPIE Proceedings. 